\title{
Experimental Observation and Theoretical Calculations of Rydberg Series in Hollow Lithium Atomic States
}

\author{
S. Diehl, ${ }^{1}$ D. Cubaynes, ${ }^{1}$ F. J. Wuilleumier, ${ }^{1}$ J.-M. Bizau, ${ }^{1}$ L. Journel, ${ }^{1}$ E. T. Kennedy, ${ }^{2}$ C. Blancard, ${ }^{3}$ L. VoKy, ${ }^{4}$ \\ P. Faucher ${ }^{4}$ A. Hibbert,${ }^{5}$ N. Berrah, ${ }^{6}$ T. J. Morgan, ${ }^{7}$ J. Bozek, ${ }^{8}$ and A. S. Schlachter ${ }^{8}$ \\ ${ }^{1}$ Laboratoire de Spectroscopie Atomique et Ionique, Université Paris Sud, Bâtiment 350, 91405-Orsay Cedex, France \\ ${ }^{2}$ School of Physical Sciences, Dublin City University, Glasnevin, Dublin 9, United Kingdom \\ ${ }^{3}$ Centres d'Etudes de Limeil-Valenton, DRIF, F-94195 Villeneuve-Saint-Georges, France \\ ${ }^{4}$ Observatoire de la Côte d'Azur, Nice, BP 229, 06304 France \\ ${ }^{5}$ Queen's University of Belfast, BT7 1NN, Belfast, United Kingdom \\ ${ }^{6}$ University of Western Michigan, Kalamazoo, Michigan 40849 \\ ${ }^{7}$ Wesleyan University, Middletown, Connecticut 06459 \\ ${ }^{8}$ Lawrence Berkeley National Laboratory, Advanced Light Source, University of California, Berkeley, California 97420
}

(Received 8 April 1997)

\begin{abstract}
Several extended Rydberg series have been experimentally identified in triply excited states of hollow lithium, by use of electron spectrometry and synchrotron radiation at the Advanced Light Source. Energies, partial cross sections, and quantum defects have also been calculated using the $R$-matrix approximation. Our results show that the two inner electrons stay in a core-excited state of given symmetry while the behavior of the third electron is mostly governed by the nuclear potential screened by the two inner electrons. [S0031-9007(97)03826-X]
\end{abstract}

PACS numbers: $32.80 . \mathrm{Hd}, 32.80 . \mathrm{Fb}, 32.80 . \mathrm{Rm}$

We present here the first experimental evidence demonstrating the existence of several extended Rydberg series in the hollow triply excited states of lithium. These new results, obtained using electron spectrometry and synchrotron radiation, are supported by our theoretical calculations made using the $R$-matrix approximation. We also explain why these Rydberg series have not been observed previously.

The inner shell $(K$, or $1 s)$ in a hollow atomic or ionic state is empty. Simultaneous excitation of all three electrons can create hollow lithium states of the type $n \ell n^{\prime} \ell^{\prime} n^{\prime \prime} \ell^{\prime \prime}$ with $n \geq 2$ [1-5]. A calculation of some low-lying hollow lithium states in hyperspherical coordinates has been recently carried out [6] as a step towards the development of a classification scheme for triply excited states, analogous to the $(K, T)^{A}$ radial and angular correlation quantum numbers $[7,8]$ used to describe the doubly excited states of helium [9]. For future understanding an important question is whether hollow lithium states exist which can be regarded as heliumlike doubly excited $\mathrm{Li}^{+}$terms plus a running outer electron or is the configuration mixing so great that Rydberg states of this type are absent? An earlier experiment [3] measuring the yield of $\mathrm{Li}^{+}$ions by photoion spectrometry did not reveal such series features. In fact, the authors specifically stated [3] "that the most striking aspect of the ion spectrum is the complete absence of any identifiable Rydberg series structure." Later measurements taking advantage of the final-state selectivity of photoelectron spectroscopy $[4,5]$ suggested, however, that some of the observed structures could be identified as first members of Rydberg series. But their effect on partial cross sections for photoionization into final $\mathrm{Li}^{+}$ionic states with one vacancy in the $1 s$ shell was very weak and no higher terms of any series were observed.

We have carried out new theoretical $R$-matrix calculations which predict the existence of eight Rydberg series between 150 and $160 \mathrm{eV}$, i.e., $1 s^{2} 2 s^{2} S+h \nu \rightarrow$ $\left[\left(2 s^{2}{ }^{1} S\right) n p\right]^{2} P,\left[\left(2 s 2 p^{3} P\right) n s \text { or } n d\right]^{2} P,\left[\left(2 p^{2}{ }^{3} P\right) n p\right]^{2} P$, $\left[\left(2 p^{2}{ }^{1} D\right) n p\right]^{2} P,\left[\left(2 s 2 p^{1} P\right) n s \text { or } n d\right]^{2} P$, and $\left[\left(2 p^{2} \times\right.\right.$ $\left.\left.{ }^{1} S\right) n p\right]^{2} P$. In Fig. 1, we show the calculated partial cross section for photoionization into the $1 s 2 \ell$ final $\mathrm{Li}^{+}$

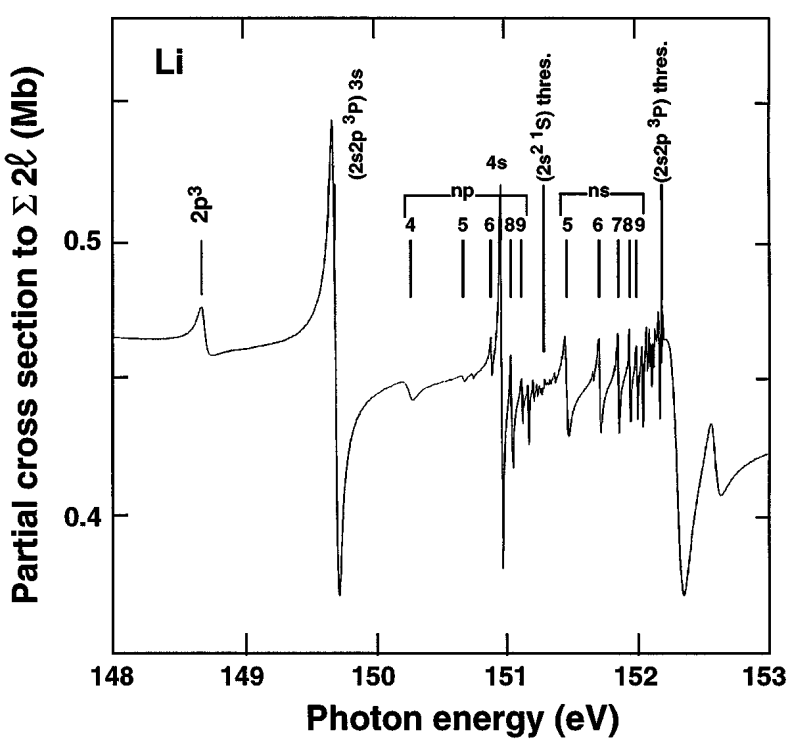

FIG. 1. $R$-matrix calculation of partial cross section for photoionization of $\mathrm{Li}$ atoms into the $(1 s 2 \ell)^{1,3} L$ final states of the $\mathrm{Li}^{+}$ion. 
ionic states from 150 to $152 \mathrm{eV}$. The series $1 s^{2} 2 s^{2} S \rightarrow$ $\left[\left(2 s 2 p^{3} P\right) n s\right]^{2} P$ is predicted to be the most intense in this energy region. For the experimental confirmation of whether such Rydberg terms exist, a key role of the calculations was the prediction of which channels they would autoionize into with a resonantly enhanced signal. A simplified energy-level diagram for $\mathrm{Li}, \mathrm{Li}^{+}$, and $\mathrm{Li}^{2+}$ is shown in Fig. 2. At excitation energies lower than the $2 s^{2}{ }^{1} S(151.66 \mathrm{eV})$, the $n=3$ and $n=4$ terms of the $\left[\left(2 s 2 p^{3} P\right) n s^{2} P\right]$ series only decay to the singly excited 1 sne states. These decay paths cause interferences with the direct photoionization route as reported earlier [5]. However, the $n \geq 5$ terms of this series lie above the $2 s^{2}{ }^{1} S$ excited state of $\mathrm{Li}^{+}$. Excitation energies greater than this limit $(n \geq 5)$ allow both decay to singly and doubly excited states of the $\mathrm{Li}^{+}$ion. Decay to the $2 s^{2}{ }^{1} S$ $\mathrm{Li}^{+}$ionic state produces emission of a very low energy electron. The $2 s^{2}{ }^{1} \mathrm{SLi}^{+}$state subsequently decays only to the ground state of the doubly charged $\mathrm{Li}^{2+}$ ion, with emission of a high-energy $(70.63 \mathrm{eV})$ easily detected electron. The probability for direct double Auger decay in the $\mathrm{Li}^{2+}$ channel is much weaker. The alternative decay processes can be summarized in the following relations:

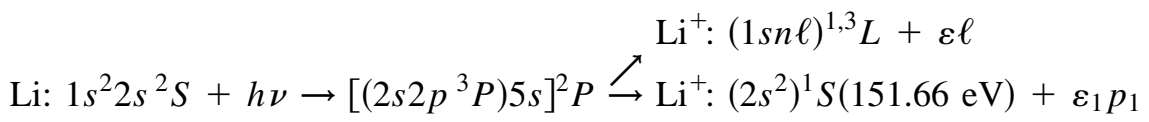

$$
\begin{aligned}
& \hookrightarrow \mathrm{Li}^{2+}: 1 s^{2} S+\varepsilon_{2} s(70.63 \mathrm{eV})
\end{aligned}
$$

As the photon energy is increased, further doubly excited $\mathrm{Li}^{+}$intermediate decay states become available. The alternative $(K, T)^{A}$ quantum numbers [7,8] of the six possible doubly excited $(n, N=2) \mathrm{Li}^{+}$ states are $2 s^{2}{ }^{1} S(1,0)^{+} ; 2 s 2 p^{3} P(1,0)^{+} ; 2 p^{23} P(0,1)^{+}$; $2 p^{21} D(1,0)^{+} ; 2 s 2 p^{1} P(0,1)^{+}$, and $2 p^{2}{ }^{1} S(-1,0)^{+}$. The

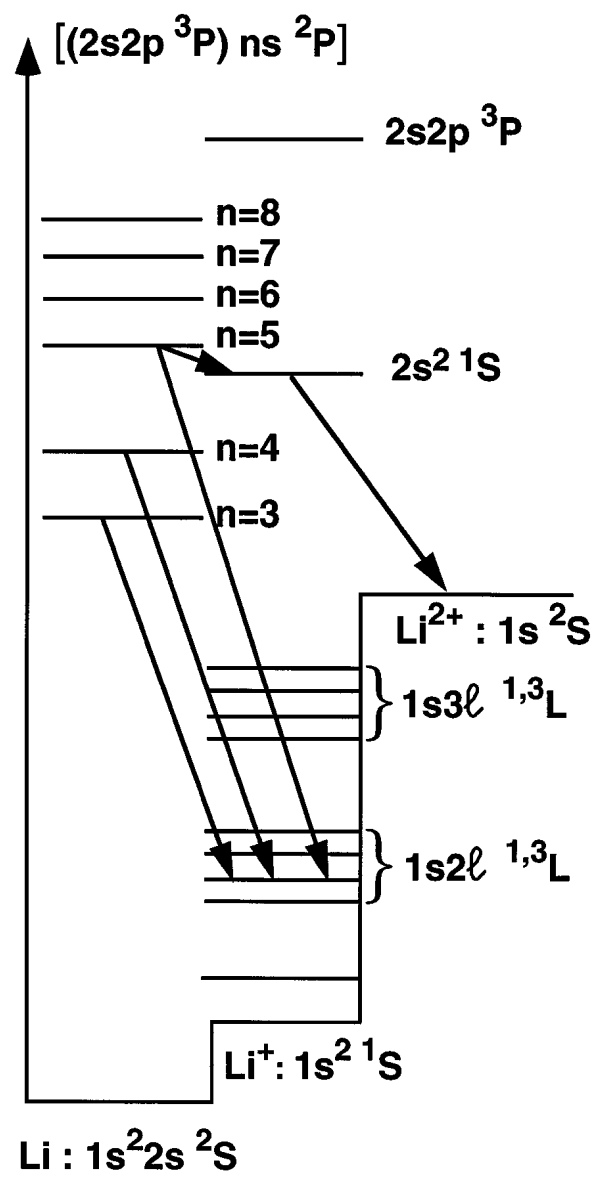

FIG. 2. Simplified partial energy-level diagram of $\mathrm{Li}, \mathrm{Li}^{+}$, and $\mathrm{Li}^{2+}$. present experiments provide, for the first time, channel selective detection of the decay of triply excited states of hollow lithium via these intermediate doubly excited $\mathrm{Li}^{+}$states, providing the required sensitivity for detecting the corresponding Rydberg series terms leading to these limits. For simplicity we will use the one-electron notation in the rest of this paper.

The present photoelectron spectrometry measurements were carried out on the undulator beam line 9.0.1 at the Advanced Light Source (ALS) in Berkeley. The experimental setup and experimental procedure have been previously described [4,5]. A spectral resolution of $40 \mathrm{meV}$ and an electron resolution $\Delta E / E$ of $0.4 \%$ were used. The relative partial cross sections were put on an absolute scale by normalization to the measured photoabsorption cross section [10]. Details about our $R$-matrix calculations have been also given elsewhere [4].

In our experiment, we measured the intensity of the high-energy electrons $\varepsilon_{2} s$ as a function of the photon energy. Since the radiative decay of a light element like $\mathrm{Li}$ is very weak compared to autoionization, the partial cross section measured from the second step of the autoionization process is essentially the cross section for photoionization into the $2 s^{2}{ }^{1} \mathrm{Li}^{+}$final ionic state. We show in Fig. 3 the results of our measurements over the excitation energy range of the $\left[\left(2 s 2 p^{3} P\right) n s\right]^{2} P$ series. The experimental data are shown in the upper part and our convoluted $R$-matrix calculations in the lower part of the figure. We present the variation of the partial cross sections, for photoionization into the singly excited level $1 s 2 s^{3} S$ (upper curve, left scale) and the doubly excited level $2 s^{2}{ }^{1} S$ (lower curve, right scale) $\mathrm{Li}^{+}$states. Autoionization of the $n=3$ and $n=4$ terms into $\mathrm{Li}^{+}\left[1 s 2 s^{3} S\right]$ has only a weak effect on the corresponding partial cross section, as expected from our $a b$ initio calculations and suggested by our earlier measurements [5]. For $n \geq 5$ the excitation energy of the $2 s^{2}{ }^{1} S$ ionization limit is exceeded and well resolved electron lines are observed in this 


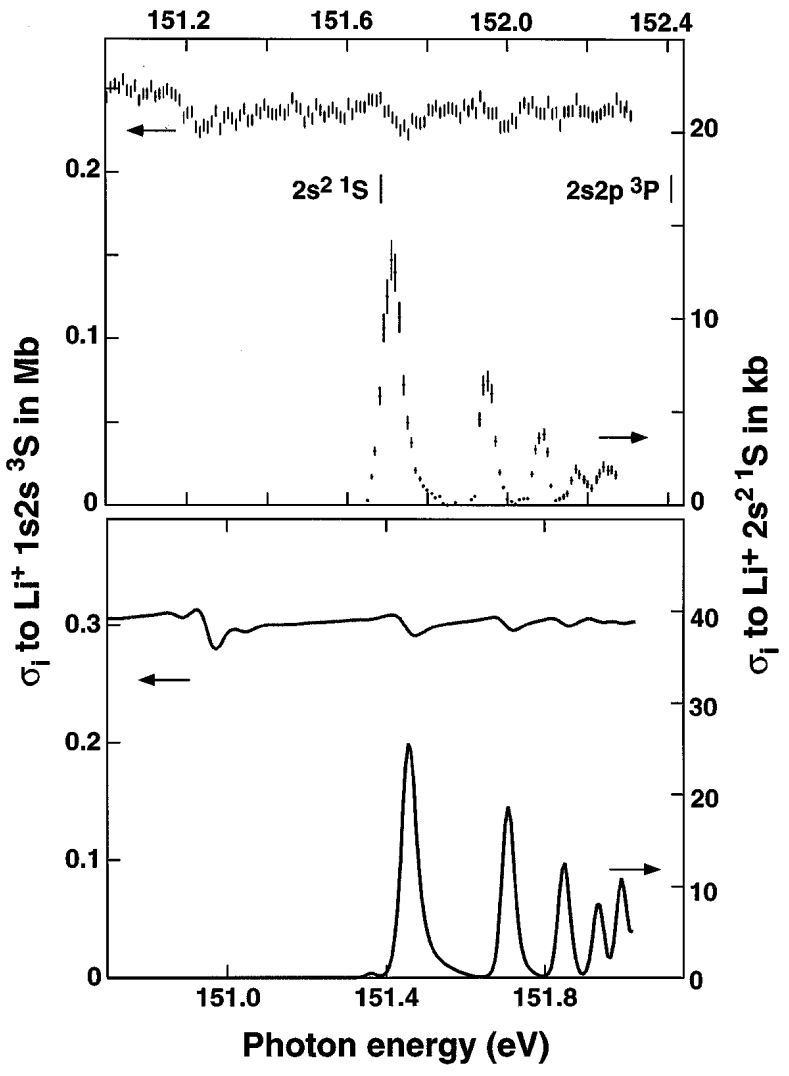

FIG. 3. Upper panel: measured values of partial cross sections for photoionization of $1 s^{2} 2 s^{2} S \mathrm{Li}$ atoms into $1 s 2 s^{3} S$ (upper curve, left scale) and $2 s^{2}{ }^{1} S$ (lower curve, right scale) $\mathrm{Li}^{+}$ ionic states. Lower panel: results of our convoluted $R$-matrix calculations for the same partial cross sections as in the upper panel.

channel with intensities decreasing with increasing $n$; Rydberg states with $n$ extending up to 8 are clearly evident. Particularly noteworthy is the contrast of the resonant Rydberg series when detected in the $2 s^{2}{ }^{1} S$ channel; the extremely low associated background ensures that they are strongly evident despite the fact that the partial cross section at the top of the most intense resonance is less than $15 \mathrm{~kb}$. The regular intensity behavior of the observed Rydberg series implies that the relaxation process which leads to ionization of the outer electron does not depend much on the running $n \ell$ orbital. The partial cross section for photoionization into the $1 s 2 s^{3} S$ is many times greater $(>200 \mathrm{~kb})$; nevertheless the Rydbergs are barely noticeable in this channel due to the large continuum arising from the direct nonresonant photoionization process. These considerations explain why such an extended Rydberg series was not observed in previous photoionization experiments detecting the ion yield in the $\mathrm{Li}^{+}$channel. They should, however, be observable in the $\mathrm{Li}^{2+}$ channel, especially between $151.66 \mathrm{eV}$ (energy of the $2 s^{2}{ }^{1} S$ threshold) and $152.41 \mathrm{eV}\left(2 s 2 p^{3} \mathrm{P} \mathrm{Li}^{+}\right.$threshold $)$: In this energy region, photoion [11] and photoelectron spectrometries should give the same results if one neglects the weak direct double photoionization process. We show in the lower part of Fig. 3 the results of our $R$-matrix calculations for the same partial cross sections. The length and velocity forms cannot be distinguished within the scale of the figure. There is excellent qualitative agreement between our experimental and theoretical data, as was already observed for photoionization into many of the $1 s n \ell^{1,3} L$ states of $\mathrm{Li}^{+}$ions [5].

We show in Fig. 4 a second example of a Rydberg series to further illustrate the quality of the experimental data and to demonstrate that extended Rydberg series are a general feature of hollow lithium states (at least with $N=2$ ). According to our calculations and to preliminary data [12], the energies of the members of the $1 s^{2} 2 s+h \nu \rightarrow\left[\left(2 p^{2}{ }^{1} S\right) n p\right]^{2} P$ series lie between 156 and $159 \mathrm{eV}$, converging to the doubly excited ionization limit $2 p^{2}{ }^{1} S$ at 159.16 (2) eV. We show the partial cross sections for decay of this series via the $2 p^{21} D$ (upper panel) and $2 s 2 p^{1} P$ (lower panel) doubly excited $\mathrm{Li}^{+}$ states, both of which lie lower in energy than the $n=3$ member of the triply excited Rydberg series. In both panels the results of our $R$-matrix calculations are shown as solid lines, after convolution with the experimental spectral resolution. They are in good agreement with the experimental data. It should be noted in this case that the theoretical cross sections are lower than the experimental values. The profiles of the cross section are predominantly symmetric and Lorentzian in the $2 p^{2}{ }^{1} D$ channel, suggesting that the interference between the direct and resonant paths is weak in this channel. The relaxation processes leading to decay in these two channels $\left(2 p^{2}{ }^{1} D\right.$ and $2 s 2 p^{1} P$ ) are different; core relaxation is required for the decay to the $2 s 2 p^{1} P$, whereas a coupling change suffices for decay to the $2 p^{21} D$.

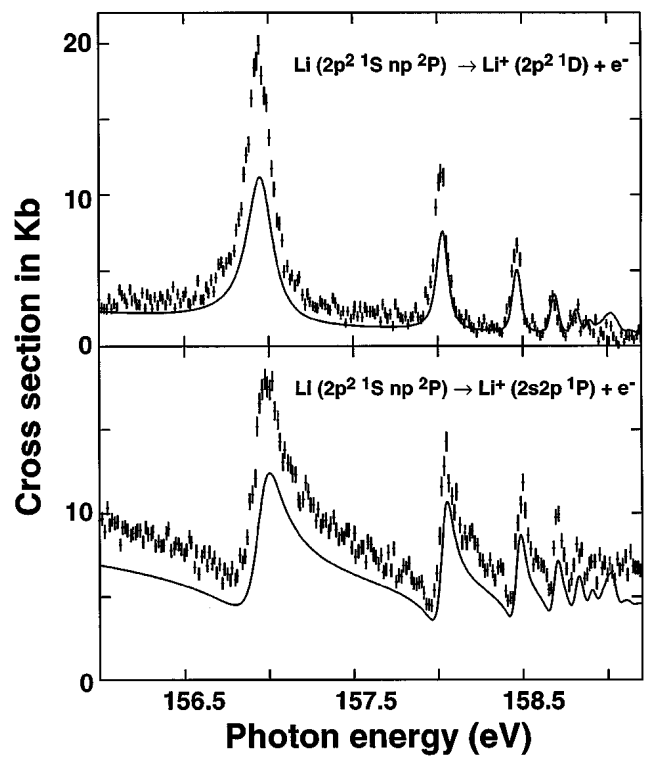

FIG. 4. Upper and lower panels show the experimental and convoluted $R$-matrix (solid line) results for photoionization of $1 s^{2} 2 s^{2} S \mathrm{Li}$ atoms into the $2 p^{2}{ }^{1} D$ and $2 s 2 p^{1} P$ ionic states of the $\mathrm{Li}^{+}$ion, respectively. 
TABLE I. Energies (in eV) and quantum defects $\delta$ of members of the $1 s^{2} 2 s^{2} S+h \nu \rightarrow\left[\left(2 s 2 p^{3} P\right) n s\right]^{2} P$ Rydberg series. The uncertainty in the experimental energies is $\pm 0.02 \mathrm{eV}$.

\begin{tabular}{lcccc}
\hline \hline & \multicolumn{2}{c}{$E_{n}(\mathrm{eV})$} & \multicolumn{2}{c}{$\delta$} \\
$n$ & Experiment & $R$-matrix & Experiment & $R$-matrix \\
\hline $3^{\mathrm{a}}$ & $149.95^{\mathrm{b}}$ & 149.69 & $0.65(2)$ & 0.66 \\
4 & $151.22^{\mathrm{b}}$ & 150.95 & $0.62(3)$ & 0.67 \\
5 & 151.68 & 151.46 & $0.68(3)$ & 0.65 \\
6 & 151.92 & 151.71 & $0.73(5)$ & 0.62 \\
7 & 152.06 & 151.85 & $0.77(6)$ & 0.58 \\
8 & 152.15 & 151.93 & $0.77(8)$ & 0.62 \\
$\infty$ & 152.41 & 152.18 & & \\
\hline \hline
\end{tabular}

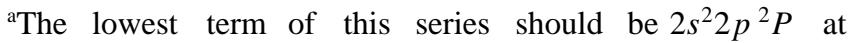
$142.25 \mathrm{eV}$. However, this state is so strongly perturbed that it cannot be considered as part of the Rydberg series. (The calculated quantum defect would be 0.84.)

${ }^{\mathrm{b}}$ Average from Refs. [2] and [5].

We present in Tables I and II our measured values for the energies of the various terms of the $\left[\left(2 s 2 p{ }^{3} P\right) n s\right]^{2} P$ and $\left[\left(2 p^{2}{ }^{1} S\right) n p\right]^{2} P$ Rydberg series, respectively, together with the results of our calculations. For the $\left[\left(2 s 2 p^{3} P\right) n s\right]^{2} P$ series the energies of the $n=3$ and $n=4$ terms were previously determined [5] from measurements into the $1 s 2 p^{1,3} P$ and $1 s 4 \ell{ }^{1,3} L$ channels. The calculated energies of the $n$ terms of this series are all lower by about $0.2 \mathrm{eV}$ and the absolute values of the calculated partial cross sections are again slightly higher than the experimental results, as was previously the case for the main hollow-lithium states [5]. (One should keep in mind, however, that the accuracy of the photoabsorption measurements is not better than 25\%.) We also determined the quantum defects for the series and compared them with the results of our $R$-matrix calculations as shown in the right part of Table I. Agreement between experimental and theoretical data is excellent for the first three terms. For the higher terms, the theoretical results seem to decrease slightly while the experimental data increase slightly; the error bars, however, are much larger for these states. The experimental and theoretical values

TABLE II. Energies (in eV) and quantum defects $\delta$ of members of the $1 s^{2} 2 s^{2} S+h \nu \rightarrow\left[\left(2 p^{2}{ }^{1} S\right) n p\right]^{2} P$ Rydberg series. The uncertainty in the experimental energies is $\pm 0.02 \mathrm{eV}$.

\begin{tabular}{lcccc}
\hline \hline & \multicolumn{2}{c}{$E_{n}(\mathrm{eV})$} & \multicolumn{2}{c}{$\delta$} \\
$n$ & Experiment & $R$-matrix & Experiment & $R$-matrix \\
\hline $3^{\mathrm{a}}$ & 156.97 & 156.97 & $0.51(1)$ & 0.52 \\
4 & 158.05 & 158.06 & $0.50(2)$ & 0.52 \\
5 & 158.50 & 158.51 & $0.46(3)$ & 0.49 \\
6 & 158.71 & 158.73 & $0.49(4)$ & 0.50 \\
7 & 158.84 & 158.86 & $0.48(6)$ & 0.48 \\
$\infty$ & 159.16 & 159.18 & & \\
\hline \hline
\end{tabular}

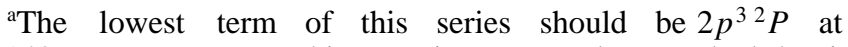
$148.7 \mathrm{eV}$. However, this state is so strongly perturbed that it cannot be considered as part of the Rydberg series. (The calculated quantum defect would be 0.82 .) of energies and quantum defects for the $\left[\left(2 p^{2}{ }^{1} S\right) n p\right]^{2} P$ Rydberg series (Table II) are in excellent agreement. The numbers for the quantum defects are remarkably constant throughout the series. Its absolute value for this series is smaller than that for the $\left[\left(2 s 2 p^{3} P\right) n s\right]^{2} P$ series as expected, since the orbital of an $n p$ electron penetrates the two core-excited electrons less than does an $n s$ electron.

To conclude, we have definitely demonstrated that many of the hollow states of atomic lithium can be classified into extended Rydberg series. The present results confirm that these hollow states can be considered to be formed of two core-excited inner electrons strongly bonded together in a given symmetry while the third electron behaves like a quasihydrogenic electron, even though in the triply excited states the one-electron orbitals are modified by the strong perturbation introduced in the atom by the absence of electrons in the $1 s$ inner shell.

The support of the Centre d'Etudes CEA de LimeilValenton (DRIF), Advanced Light Source, EU Human and Mobility Program under Contract No. 93-0361, Forbairt International Collaboration Scheme, and DOE (BES) under Contract No. DRE-FG02-92ER14299 is gratefully acknowledged.

[1] L. M. Kiernan, E. T. Kennedy, J.-P. Mosnier, J. T. Costello, and B.F. Sonntag, Phys. Rev. Lett. 72, 2359 (1994).

[2] L. M. Kiernan, M.-K. Lee, B. F. Sonntag, P. Sladeczek, P. Zimmermann, E. T. Kennedy, J.-P. Mosnier, and J. T. Costello, J. Phys. B 28, L161 (1995).

[3] Y. Azuma, S. Hasegawa, F. Koike, G. Kutluk, T. Nagata, E. Shigemasa, A. Yagishita, and I. A. Sellin, Phys. Rev. Lett. 74, 3770 (1995).

[4] L. Journel, D. Cubaynes, J.-M. Bizau, S. Al Moussalami, B. Rouvellou, F. J. Wuilleumier, L. VoKy, P. Faucher, and A. Hibbert, Phys. Rev. Lett. 76, 30 (1996).

[5] S. Diehl, D. Cubaynes, J.-M. Bizau, L. Journel, B. Rouvellou, S. Al Moussalami, F. J. Wuilleumier, E. T. Kennedy, N. Berrah, C. Blancard, T. J. Morgan, J. Bozek, A. S. Schlachter, L. VoKy, P. Faucher, and A. Hibbert, Phys. Rev. Lett. 76, 3915 (1996).

[6] X. Yang, C. G. Bao, and C. D. Lin, Phys. Rev. Lett. 76, 3096 (1996).

[7] D. R. Herrick and O. Sinagoglu, Phys. Rev. A 11, 93 (1975).

[8] C. D. Lin, Phys. Rev. A 29, 1019 (1984).

[9] K. Schulz, G. Kaindl, M. Domke, J. D. Bozek, P. A. Heimann, A. S. Schlachter, and J. M. Rost, Phys. Rev. Lett. 77, 3086 (1996).

[10] G. Mehlman, J. W. Cooper, and E. B. Saloman, Phys. Rev. A 25, 2113 (1982).

[11] Y. Azuma, S. Kasegawa, F. Koike, G. Kutluk, T. Nagata, E. Shigemasa, A. Yagishita, and A. Sellin, in Atomic and Molecular Photoionization, edited by A. Yagishita and T. Sasaki (Un. Ac. Press, Tokyo, 1996), p. 253.

[12] F. J. Wuilleumier, S. Diehl, D. Cubaynes, and J. M. Bizau, in X-Ray and Inner-Shell Processes, edited by R.L. Johnson, H. Schmidt-Böcking, and B.F. Sonntag, AIP Conf. Proc. No. 389 (AIP, New York, 1997), p. 625. 